\title{
Machine Learning Methods for Automated Detection of Severe Diabetic Neuropathy
}

\author{
Herbert F Jelinek ${ }^{1,2^{*}}$, David J Cornforth ${ }^{2}$ and Andrei V Kelarev ${ }^{1}$ \\ ${ }^{1}$ Centre for Research in Complex Systems and School of Community Health, Charles Sturt University, Albury, NSW, Australia
}

${ }^{2}$ Applied Informatics Research Group, University of Newcastle, University Drive, Callaghan, NSW 2308, Australia

"Corresponding author: Herbert F Jelinek, Centre for Research in Complex Systems and School of Community Health, Charles Sturt University, Albury, NSW, Australia, Tel: +61-2-6051-9219; E-mail: hjelinek@csu.edu.au

Rec date: April 18, 2016; Acc date: May 17, 2016; Pub date: May 24, 2016

Copyright: ( 2016 Jelinek HF, et al. This is an open-access article distributed under the terms of the Creative Commons Attribution License, which permits unrestricted use, distribution, and reproduction in any medium, provided the original author and source are credited.

\begin{abstract}
Objective: The present study aimed at investigating machine learning methods for automated detection of severe diabetic neuropathy. Severe diabetic neuropathy represents a significant neurological problem in diabetes as it requires urgent intervention to reduce the risk of sudden cardiac death. Automated detection provides a tool that can be applied to clinical data and for identifying comorbidities that can trigger diagnosis and treatment.

Methods: We applied multi scale Allen factor to determine heart rate variability, a marker for diabetic neuropathy from ECG recordings as features to be used for the machine learning methods and automated detection. The major innovation of this work is the introduction of a new Graph-Based Machine Learning System (GBMLS). This method is intended to enhance the effectiveness of the diagnosis of severe diabetic neuropathy. We applied it to the multi scale Allen factor (MAF) features as a collection of attributes determined from the recorded ECG bio signals. These attributes can be collected as a result of routine ECG investigation of patients regardless of the presenting medical problems.
\end{abstract}

Results: Our experiments compared the sensitivity and specificity of the automated detection produced by GBMLS with analogous outcomes achieved by various other machine learning approaches. To this end we used a comprehensive collection of important classifiers and clusterers available in the open source machine learning software package Scikit-learn. The experiments have demonstrated that the best outcomes were obtained by GBMLS in combination with MAF, which improved sensitivity to 0.89 and specificity to 0.98 and outperformed several other classifiers and clusterers including Random Forest with sensitivity of 0.83 and specificity of 0.92 .

Conclusion: The novel GBMLS machine learning technique applied to MAF attributes has outperformed other machine learning methods and achieved excellent sensitivity and specificity. These results are significant and sufficiently effective to be recommended for practical application of this technique.

Keywords: Automated detection; Classifiers; Heart rate variability; Graph representation; Machine learning; Multi scale allen factor; Severe diabetic neuropathy

\section{Introduction}

Diabetic neuropathy associated complications, which affects all major organs of the body, are common in Type 1 and Type 2 diabetes $[1,2]$. Cardiac diabetic neuropathy is characterised by damage to nerves regulating the heart rate and any changes in the capacity of these nerves to modulate heart rate leads to changes to heart rate variability (HRV). Its prevalence lies between $20 \%$ and $60 \%$ in patients with diabetes, with a mortality that is approximately five times higher $[3,4]$.

Testing for cardiac autonomic neuropathy (CAN) in people with diabetes was traditionally based on five Ewing cardiac reflex tests that constitute the gold standard. Recent research has been investigating the efficacy of alternative diagnostic tests, using ECG attributes [5-8] to address shortcomings of the Ewing battery as a number of the Ewing tests included in the Ewing battery are often counter-indicated for patients with cardiorespiratory comorbidity, frail or severely obese patients [9]. Therefore resting supine recordings of ECG that provide heart rate information may be better suited for this clinical population and may be more sensitive and accurate. A number of previous studies have shown the effectiveness of HRV attributes for classifying cardiac pathology [10-12] for more details and further references). Heart rate variability is also decreased in diabetes mellitus type 1 and diabetes type 2 . The majority of research has concentrated on investigating the time and frequency domain attributes with some more recent work reporting findings for nonlinear HRV attributes [13-19].

The current research investigated the application of HRV attributes and advanced data mining systems in improving identification of severe diabetic neuropathy. Previously, high levels of accuracy in the diagnosis of mild and moderate stages of CAN have been achieved by classifiers proposed by Kelarev et al. [20-26]. However, these experiments included the original Ewing test results in their classifiers. In contrast, the present paper proposes a system for automated diagnosis of severe diabetic neuropathy on the basis of multi scale Allan factor attributes that can be easier collected compared to the routine collection of Ewing test results. 
This is the first article concentrating on automated detection of severe diabetic neuropathy as defined the battery classification by Ewing et al. [27,28]. Severe diabetic neuropathy is characterised by both sympathetic and parasympathetic dysfunction that contributes to the reported increased risk of arrhythmias and sudden cardiac death. This makes detection of severe diabetic neuropathy a priority and requires accurate bio signal analysis and classification algorithms to improve identification of at risk patients and treatment effectiveness [3]. The present article applies a novel clustering approach based on graphs (GBMLS) classifier for the identification of severe diabetic neuropathy using multi scale Allan factor (MAF). For complete definition of MAF, we refer to Section 2.5.1 by Teich et al. [29]. Our experiments carefully compare the results of GBMLS system with traditional data mining algorithms. We hypothesise that GBMLS system improves the effectiveness of identifying severe diabetic neuropathy when combined with MAF obtained from the heart rate bio signal.

Background information on previous graph-based methods and further details on the diagnosis of severe diabetic neuropathy, preliminaries on Graph-Based Machine Learning System (GBMLS) methodology, CAN pathophysiology, the diabetes health screening database (DiScRi/DiabHealth) and HRV analysis are given in the next sections.

\section{Brief review of literature and preliminaries}

The Ewing battery is the traditional clinical assessment tool for CAN and CAN severity $[27,28]$. From the five Ewing test results, three measure parasympathetic activity (lying to standing heart rate change, Valsalva manoeuvre, changes in heart rate with rhythmic breathing) and two sympathetic activity (lying to standing blood pressure change, and diastolic blood pressure change with hand grip). For severe diabetic neuropathy two of the parasympathetic tests and any one of the sympathetic tests need to be abnormal [28].

Diabetic neuropathy can be investigated by assessing cardiac rhythm function and cardiac rhythm pathology. Cardiac autonomic neuropathy $(\mathrm{CAN})$ is a pathology that clusters within the umbrella of diabetic neuropathy and assessing cardiac rhythm is the most suitable clinical tool for identification of diabetic neuropathy. This is the first article concentrating on the identification of severe diabetic neuropathy. Our experiments investigated two classes: severe diabetic neuropathy and absence of severe diabetic neuropathy. The class of severe diabetic neuropathy has not been investigated previously as a paradigm in machine learning classification. The absence of severe diabetic neuropathy or complement is a union of three classes considered previously in the literature: no CAN, early CAN, definite CAN, see $[27,28]$ for more explanations and details. When we focus on just one class of severe diabetic neuropathy and combine all other classes into their union, it is possible to achieve higher effectiveness in solving the task of identifying only severe diabetic neuropathy and ignoring differences between other classes, which clinically are less dangerous to the patients [29].

Cardiac autonomic neuropathy relates to abnormal cardiac rhythm that increases risk of sudden cardiac death, especially in diabetes mellitus type 1 and type $2[30,31]$. Although a number of invasive and non-invasive assessments can be made that identify diabetes such as blood glucose levels, retinal photography and plantar foot pressure, these tests do not identify cardiac autonomic neuropathy specifically [32-35].
Previous work on data mining and HRV analysis concentrated mainly on standard time and frequency-domain methods and applied automated machine learning assessment of the original Ewing categorization of CAN using HRV attributes [35-37]. However, data mining methods applied to HRV data similar to MAF have been reported by Cornforth et al. [38,39], who examined the effectiveness of 80 time and frequency-domain attributes for the detection of the early stages of CAN, concentrating on the applications of genetic algorithms when searching for a subset of HRV attributes that are optimal for the detection of early CAN.

The motivation to use HRV data is that it is richer than the results of the five Ewing tests and more often and easier to obtain in clinical practice than the Ewing battery tests.

\section{Methodology Applied to Collect Data}

In order to investigate the role of HRV attributes and the capability of multi-level clusterers in improving diagnostic accuracy, a large database of test results and health-related parameters collected through the Charles Sturt Diabetes Complications Screening Group (DiScRi/ DiabHealth), containing Ewing battery results and HRV data was selected [40].

Participants were recruited as part of the Charles Sturt University Diab Health screening [41]. Clinical and demographic data as well as the Ewing battery results and ECG records were obtained during 2011-2014. The university human research ethics committee provided consent for the study and all participants gave informed consent following an information session prior to recording any subjects. All participants had to be free of cardiovascular, respiratory and renal disease as well as depression, schizophrenia and Parkinson's disease, which are known to alter HRV results.

Recordings were obtained with participants in a supine position following a 20-minute rest period. The same conditions were used for each participant, including a temperature stable environment, and all participants were comparable for age, gender, and resting heart rate. The 20-minute ECGs were recorded with a lead II configuration using a Mac lab Pro with Chart 7 software (AD Instruments, Sydney). The sampling rate was set to 400 samples/sec and recordings were preprocessed according to the methods described by Wessel et al. [42]. The status of CAN was defined using the Ewing battery criteria [28]. For each recording, a 15-minute segment was selected from the middle in order to remove start up artefacts and movement at the end of the recording. From this shorter recording, the RR intervals were extracted. HRV analysis involves determining the inter beat intervals between successive pulses of the heart. In terms of ECG curve, these intervals are equal to the intervals between successive QRS complexes on an ECG or the intervals between the fiducial points of the successive $\mathrm{R}$ waves (RR intervals), cf. [43] and [4] for more explanations. The RR interval series for each participant was preprocessed using adaptive pre-processing and the measures used were determined from these data [42] for more details on adaptive filtering and pre-processing. The pre-processed data was then utilized to infer MAF values, as explained by Teich et al. [29].

\section{Graph-based machine learning system}

Clustering is an automated process that attempts to assign data to a number of groups referred to as clusters. The groups are not defined beforehand, but are obtained as an output of the clustering process. Here we investigate clustering algorithms for the diagnosis of severe 
diabetic neuropathy, and hence are only concerned with clustering of $\mathrm{k}=2$ groups or clusters. Every clustering algorithm considered in this paper, takes the number $\mathrm{k}$ of clusters to be produced as an input parameter, and creates a partition with precisely $\mathrm{k}$ groups or clusters.

To obtain a stable and reliable partition, we introduce a new clustering algorithm - Graph-Based Machine Learning System (GBMLS). GBMLS uses a novel model involving a new graph representation. Here we are using standard notation and terms of the graph theory as, for instance, in the monograph [44] and the article [45].

Let $p$ be the number of all patients from the Diab Health database with ECG data used in our experiments. In order to divide these patients into groups, we used several independent partitions and then combined their outcomes by applying our new GBMLS procedure. Our experiments compared the effectiveness of the GBMLS algorithm with several other methods presented in the next sections. In the remainder of this section, we discuss the GBMLS algorithm for the case of two clusters considered in the current experiments.

To begin the operation of GBMLS algorithm, we generated a collection of 80 independent random initial partitions

$$
K=\left\{C_{1}, C_{2}, \ldots, C_{80}\right\}(1)
$$

They were generated using the K Means [46] and Mean Shift [47] clustering algorithms, implemented in the open source machine learning package Scikit-learn et al. $[48,49]$. To make sure that the collection (1) consists of independent random partitions generated using initial points covering the space optimally, we ran each of these two clustering algorithms for 40 random values of their input parameters to obtain 40 different random partitions for each clusterer. Then we combined all of these partitions into the common collection $\mathrm{K}$ of 80 partitions.

More specifically, the output of the Mean Shift algorithm depends on the value of the input parameter 'seeds', which is used to initialise the iteration process. We ran Mean Shift with 40 random values of the 'seeds' to ensure that it creates 40 different and independent output partitions. Likewise, the output of K Means algorithm depends on the selection of initial centroids. It is determined by the input parameters 'init' and 'random state'. We set the value of 'init' equal to the string 'random'. This option makes K Means start with a set of initial centroids chosen randomly in the data set. The random selection process of initial centroids in KMeans depends on the value of the 'random state' parameter, which is used every time as a seed to the random number generator incorporated in the algorithm. We ran $\mathrm{K}$ Means 40 times with 40 different random values of the 'random state' parameter to make sure that it generated independent random partitions.

This means that for each value of $=1,2, \ldots, 80$, each particular partition is made up of two groups or clusters:

$$
C_{j}=\left\{C_{j 1}, C_{j 2}\right\}(2)
$$

which partition the set of patients so that the whole set is a disjoint union of these clusters

$$
X=C_{j 1} \cup C_{j 2}(3)
$$

GBMLS procedure takes the collection of 80 partitions as input and produces a new common partition

$$
C=\left\{C_{1}, C_{2}\right\}(4)
$$

which also divides the whole set of patients

$$
X=C_{1} \cup C_{2}(5)
$$

and at the same time achieves the best agreement with all the given partitions .

GBMLS procedure uses a graph $G=(V, E)$ with the set $\mathrm{V}$ of vertices and the set $\mathrm{E}$ of edges. The collection of vertices of the graph is a union of three subsets $V=Y \cup Z$. The number of vertices in the first subset $Y$ is equal to the number $\mathrm{p}$ of patients, so that $Y=\left\{u_{1}, u_{2}, \ldots, u_{p}\right\}$. For $=1,2, \ldots, \mathrm{p}$, the vertex corresponds to the patient. The number of vertices in the second subset is equal to $2 \times 80$ $=160$, the number of groups or clusters in all partitions , , ..., . This means that $Z=\left\{u_{p+1}, u_{p+2}, \ldots, u_{p+160}\right\}$, with vertices representing the respective groups or clusters. The collection of edges of the graph is in turn a union of three subsets: $E=\mathrm{D} \cup F \cup H$. The set $\mathrm{D}$ contains all edges of the form $\left(u_{j_{1}}, u_{j_{2}}\right)$, for all positive integers $j_{1}, j_{2}$ satisfying inequalities $1 \leq j_{1}<j_{2} \leq p$. The weight of this edge is set equal to the number of clusters where the patients belong together. The set F comprises of all edges of the form

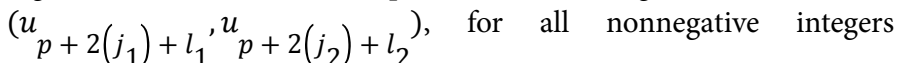
$j_{1}, j_{2}, l_{1}, l_{2}$ satisfying inequalities $0 \leq j_{1}<j_{2} \leq 79$ an $\mathrm{D}$ $1 \leq l_{1}, l_{2} \leq 2$. This edge represents the following pair of clusters: the cluster $C_{\left(j_{1}+1\right) l_{1}}$ of the partition $C_{\left(j_{1}+1\right)}$ and the cluster $C_{\left(j_{1}+1\right)}$ of the partition $C_{\left(j_{2}+1\right)}$. The weight of this edge is set equal to the well-known Jaccard similarity index $w\left(C_{\left(j_{1}+1\right) l_{1}}, C_{\left(j_{2}+1\right)} l_{2}\right)$, which measures the similarity of the clusters $C_{\left(j_{1}+1\right) l_{1}}$ and $C_{\left(j_{2}+1\right) l_{2}}$. Finally, the set contains all edges $\left(u_{i}, u_{p+2(j)+l}\right)$, for all nonnegative integers, , satisfying inequalities $1 \leq i \leq p, 0 \leq j \leq 79$, $1 \leq l \leq 2$ where the patient corresponding to the vertex $u_{i}$ belongs to the group $C_{j l}$ of the partition $C_{j}$. To partition the graph into two clusters we used freeware METIS library for partitioning of graphs described by Karypis et al. [50]. The final outcome of GBMLS is then given by the way METIS partitions all vertices of the set corresponding to the patients. Note that the graph $G=(V, E)$ is neither complete nor bipartite.

\section{Other machine learning systems}

Our experiments compared the performance of GBMLS with the following machine learning systems available in open source package by Scikit-learn et al. [49]: K Means [46], Mean Shift [47], Birch [50,51], DBSCAN [52] and Ward hierarchical clustering [53] for the diagnosis of severe diabetic neuropathy.

Furthermore, in addition to the comparisons mentioned above we have also studied the effectiveness of the GBMLS algorithm in comparison to the Hybrid Bipartite Graph Formulation (HBGF) and Cluster-Based Graph Formulation (CBGF) defined by Fern et al. [54]. These algorithms rely on different and substantially smaller graphs. 
The number of edges and vertices in these graphs and their architecture are different from the graph utilized in GBMLS. Let us refer to [55] for previous work in other research domains using HBGF and CBGF algorithms and more detailed bibliography. Moreover, our experiments compared the outcomes obtained by GBMLS with the results produced by numerous other clusterers and classifiers available in the open source machine learning package Scikit-learn et al. [48,49]. This section presents classifiers and clusterers being compared with GBMLS.

Decision Tree (DT) is a decision tree classifier using an optimised version of the Classification and Regression Trees (CART) algorithm. CART is similar to C4.5 classifier. However, it is capable of handling both classification and regression, and unlike C4.5 algorithm, it does not compute rule sets.

Three versions of the Naive Bayes algorithm were also applied from the Scikit-learn package being Gaussian Naive Bayes (GNB), Multinomial Naive Bayes (MNB), and Bernoulli Naive Bayes (BNB).

Nearest Centroid (NC) [48,49] is a classifier using classes determined by centroids similar to the clusters of the classical k-means clusterer.

Further, we have investigated Support Vector Machines (SVM). Scikit-learn includes two versions of SVM: named SVM and NuSVM. They can operate with the following kernels: 'linear', 'poly', 'rbf', 'sigmoid'. To indicate the kernel being invoked we use the following notation: SVM(linear), SVM(poly), SVM (rbf), SVM(sigmoid), NuSVM (linear), NuSVM (poly), NuSVMC (rbf), NuSVM (sigmoid).

There are also two versions of the nearest neighbour classifier in Scikit-learn [49]: K Neighbors (KN) and Radius Neighbors (RN). KN applies nearest neighbours. RN applies all neighbours contained in a sphere of radius indicated by the user as a parameter. For DiScRi data, $\mathrm{RN}$ algorithm produced substantially worse outcomes than $\mathrm{KN}$, and so we did not include $\mathrm{RN}$ in the diagrams below.

Random Forest (RF) is an efficient ensemble classifier. It operates using one of two criteria measuring the quality of split of data: Gini Impurity or Information Gain. In our experiments Gini Impurity produced better outcomes, and so we include only RF with Gini Impurity in the diagrams. In the diagrams representing the results of our experiments these versions of RF are denoted by RFG and RFE, respectively. Furthermore, the number of trees in the forest can also be specified as the n_estimators parameter. In the diagrams representing the results of our experiments RF with the number of trees equal to $t$ is denoted by RF (t).

For theoretical prerequisites and more detailed information on these classifiers the readers are referred to [49] and [56].

\section{Experimental Results and Discussion}

We applied our new clustering approach GBMLS and compared it with HBGF, CBGF and with other classification and clusterers available in the machine learning package Scikit-learn et al. $[48,49]$. In testing the effectiveness of algorithms during our experiments, for each classifier we determined the sensitivity and specificity [56] for explanations of these notions. These measures are often used in medical studies to evaluate the effectiveness of classifiers. Sensitivity is the proportion of positives (patients with severe diabetic neuropathy) that are identified correctly. It is also called the True Positive Rate (TPR). Specificity is defined as the proportion of people without severe diabetic neuropathy who have a negative test result. It is also called the True Negative Rate (TNR). The values of sensitivity and specificity belong to the interval from 0 to 1 , where the best value is 1 in terms of all of these metrics.

Diab Health is the largest known database with CAN information. It is the only database available for the authors of this paper. We selected a subset with 21 patients with severe diabetic neuropathy and 232 number of instances. All these instances contained complete HRV attributes in the Diab Health database. We recorded all available demographic and clinical parameters for these patients in a csv file. To prepare data for the experiments we added MAF values.

In order to compare the performance of GBMLS with other systems available in the Scikit-learn et al. [49], we had to determine the best kernels to be used for SVM and Nu SVM, and the best values of input parameters for several other systems for diagnosing severe diabetic neuropathy. In Scikit-learn, SVM and Nu SVM are available with four kernels: linear kernel, polynomial kernel, rbf kernel and sigmoid kernel. This means that each algorithm SVM and Nu SVM can be executed invoking any of the four kernels. We refer to [49] for more information on the formulas used in these kernels and denote these versions of SVM and Nu SVM by SVM(linear), SVM(poly), SVM (rbf), SVM(sigmoid), Nu SVM(linear), Nu SVM(poly), Nu SVM (rbf), and $\mathrm{Nu} \operatorname{SVM}($ sigmoid), respectively. First, we conducted tests to determine the performance of SVM and Nu SVM with the various available kernels. Sensitivity and specificity obtained during this first set of experiments are presented in Figure 1.

Figure 1 shows that in the diagnoses of severe diabetic neuropathy the best sensitivity and specificity were achieved by SVM with polynomial kernel. This outcome will be used to compare to the outcomes obtained by other systems below.

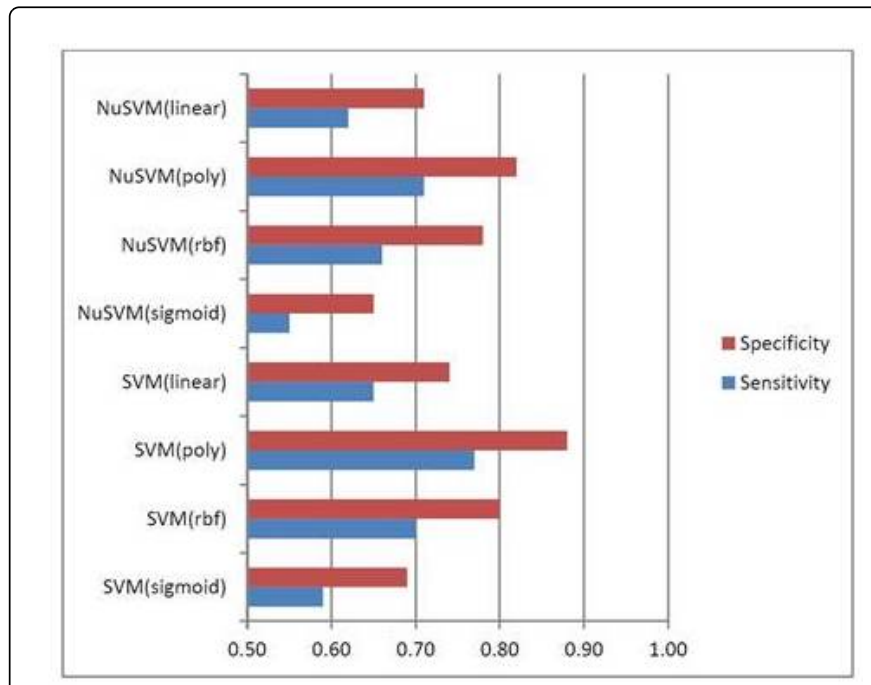

Figure 1: Sensitivity and specificity of the diagnosis of severe diabetic neuropathy by SVM and NuSVM with various kernels.

The $\mathrm{KN}$ algorithm has an input parameter $\mathrm{c}$, which is an integer specifying a fixed number of nearest neighbours to be used in the algorithm. We used the notation $\mathrm{KN}(\mathrm{c})$ to indicate the value $\mathrm{c}$ as a parameter in the diagrams representing the results of our experiments. Figure 2 presents sensitivity and specificity of the diagnosis of severe 
Citation: Jelinek HF, Cornforth DJ, Kelarev AV (2016) Machine Learning Methods for Automated Detection of Severe Diabetic Neuropathy. J

Page 5 of 7

diabetic neuropathy obtained by $\mathrm{KN}(\mathrm{c})$ for various values of the parameter $\mathrm{c}$ based on KD Tree with uniform weights.

The best sensitivity and specificity were obtained by $\mathrm{KN}$ with $\mathrm{k}=4$ (Figure 2). This result is included as the performance of $\mathrm{KN}$ in the combined diagram below.

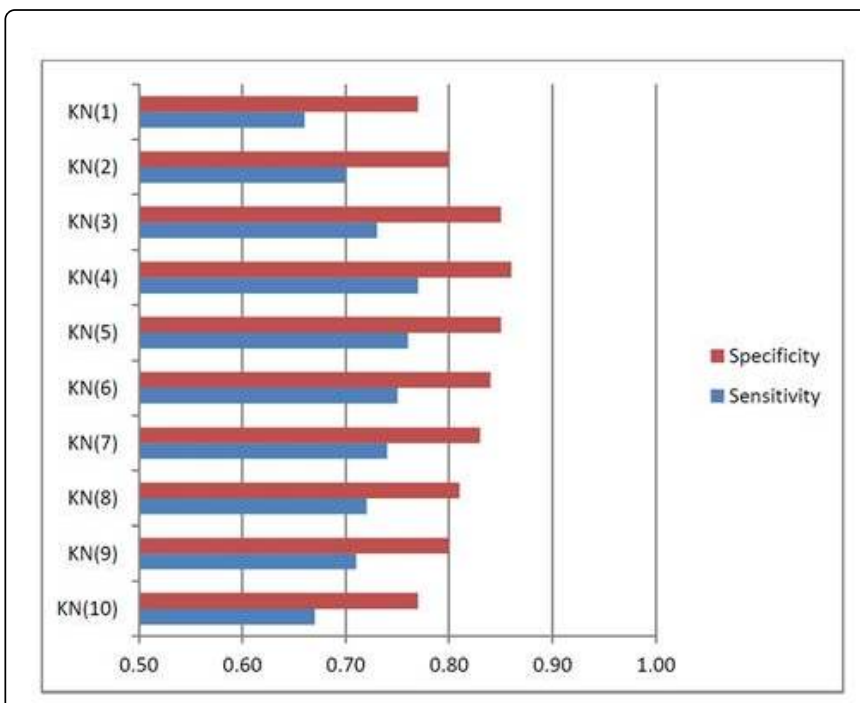

Figure 2: Sensitivity and specificity of the diagnosis of severe diabetic neuropathy by $\mathrm{KN}$.

Next, we evaluated the performance of RF for various options of the input parameter - the number of random trees. The values of sensitivity and specificity obtained are presented in Figure 3.

Figure 3 shows that the best values of sensitivity and specificity were achieved by RF using six random trees. Therefore this option is the one indicated for RF in the combined diagram below.

Finally, the results of comparing GBMLS to other clusterers CBGF, HBGF and classifiers available in the machine learning package Scikitlearn et al. [49] are depicted in Figure 4. In this diagram we included the best available options determined above for the SVM, KN and RF algorithms.

The results of all experiments show that the best sensitivity 0.98 and specificity 0.89 was obtained by the GBMLS algorithm. Note that the ensemble classifier RF implemented in the open source machine learning package Scikit-learn achieved the best performance among all classifiers and clusterers. It can therefore be recommended for clinical assessment and the diagnosis of severe diabetic neuropathy. Results of single HRV attributes do not have sufficient accuracy for classifying severe cardiac neuropathy and an algorithm is required that combines time and frequency domain as well as nonlinear HRV attributes for diagnosis [57]. GBMLS is a novel data mining algorithm that improved the accuracy for classification of severe cardiac autonomic neuropathy based on HRV attributes and make it useful in clinical practice. The algorithm combines results from time and frequency domain, and nonlinear HRV attributes to identify severe cardiac autonomic neuropathy.

Future work will be investigating several data reduction algorithms combined with GBMLS to further delineate subcategories of diabetic neuropathy into early, definite and severe CAN and provide a better basis for treatment decisions in clinical practice.

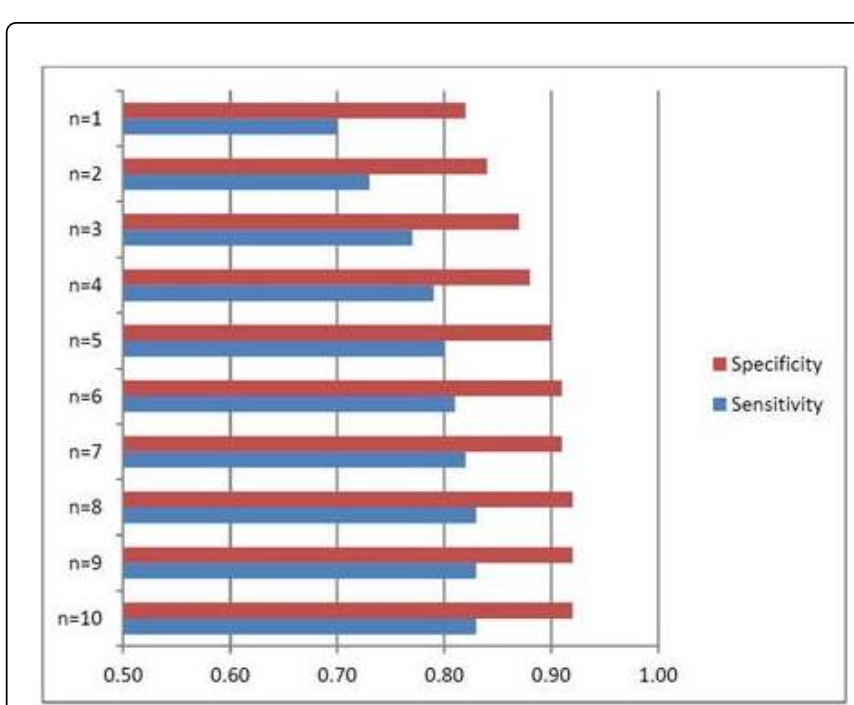

Figure 3: Sensitivity and specificity of the diagnosis of severe diabetic neuropathy by RF using various numbers of random trees.

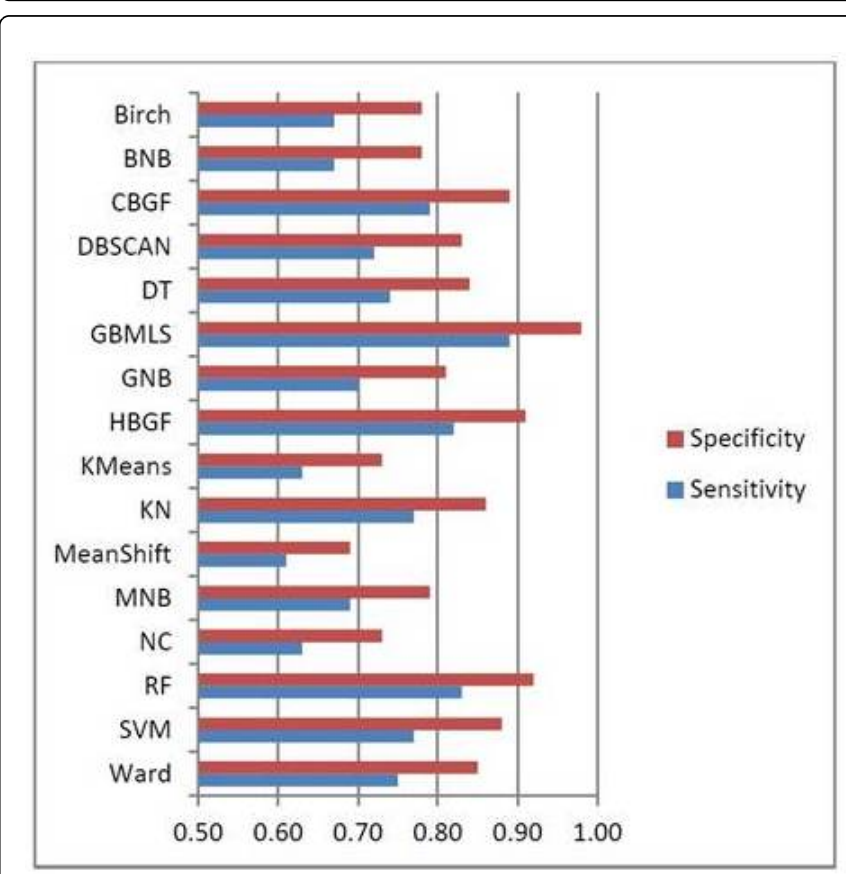

Figure 4: Sensitivity and specificity of the diagnosis of severe diabetic neuropathy by GBMLS and other machine learning systems.

\section{Conclusion}

This is the first paper concentrating on the diagnosis of severe diabetic neuropathy. It advances a new algorithm (GBMLS) for the automated detection of severe diabetic neuropathy. Our experiments compared the sensitivity and specificity of the results obtained by GBMLS to the outcomes produced by other machine learning algorithms. The results demonstrate that our new procedure outperformed other techniques and obtained the best outcomes. The 
diagnosis of severe diabetic neuropathy by GBMLS achieved the best performance level with sensitivity of 0.98 and specificity of 0.89 .

\section{Acknowledgement}

The authors wish to acknowledge the technical assistance of Bev de Jong and Paul Reidler for collecting the Ewing data. The authors would like to express their gratitude to the anonymous reviewer for comments, which have helped to improve the article.

\section{References}

1. Vinik AI, Maser RE, Mitchell BD, Freeman R (2003) Diabetic autonomic neuropathy. Diabetes Care 26: 1553-1579.

2. Witzel II, Jelinek HF, Khalaf K, Lee S, Khandoker AH, et al. (2015) Identifying Common Genetic Risk Factors of Diabetic Neuropathies. Front Endocrinol (Lausanne) 6: 88.

3. Vinik AI, Erbas T, Casellini CM (2013) Diabetic cardiac autonomic neuropathy, inflammation and cardiovascular disease. J Diabetes Investig 4: 4-18.

4. TFESC/NASPE (1996) Heart rate variability. Standards of measurement, physiological interpretation, and clinical use. Task Force of the European Society of Cardiology and the North American Society of Pacing and Electrophysiology. Euro Heart J 17: 354-381.

5. Thayer JF, Yamamoto SS, Brosschot JF (2010) The relationship of autonomic imbalance, heart rate variability and cardiovascular disease risk factors. Int J Cardiology 141: 122 -131.

6. Karmakar CK, Khandoker AH, Jelinek HF, Palaniswami M (2013) Risk stratification of cardiac autonomic neuropathy based on multi-lag ToneEntropy. Medical \& Biological Engineering \& Computing 51: 537-546.

7. Tan CO (2013) Heart rate variability: are there complex patterns? Front Physiol 4: 165.

8. Imam MH, Karmakar C, Khandoker AH, Jelinek HJ (2014) Analysing cardiac autonomic neuropathy in diabetes using electrocardiogram derived systolic-diastolic interval interactions. Computing in Cardiology 41: 85-88.

9. Baron R, Ewing DJ (1999) Heart rate variability. In: Recommendations for the Practice of Clinical Neurophysiology: Guidelines of the International Federation of Clinical Physiology. Deuschlm, A. Eisen (Eds.) Amsterdam, Elsevier.

10. Spallone V, Menzinger G (1997) Diagnosis of cardiovascular autonomic neuropathy in diabetes. Diabetes 46: S67-76.

11. Jelinek HF, Pham P, Struzik ZR, Spence I (2007) Short term ECG recording for the identification of cardiac autonomic neuropathy in people with diabetes mellitus. Proceedings of the 19th International Conference on Noise and Fluctuations, Tokyo, Japan.

12. Khandoker AH, Weiss DN, Skinner JE, Anchin JM (2011) PD2i heart rate complexity measure can detect cardiac autonomic neuropathy: an alternative test to Ewing battery. Computing in Cardiology 38: 525-528.

13. Colhoun HM, Francis DP, Rubens MB, Underwood SR, Fuller JH (2001) The association of heart-rate variability with cardiovascular risk factors and coronary artery calcification: a study in type 1 diabetic patients and the general population. Diabetes Care 24: 1108-1114.

14. Jelinek HF, Md Imam H, Al-Aubaidy H, Khandoker AH (2013) Association of cardiovascular risk using non-linear heart rate variability measures with the framingham risk score in a rural population. Front Physiol 4: 186.

15. Liao D, Carnethon M, Evans GW, Cascio WE, Heiss G (2002) Lower heart rate variability is associated with the development of coronary heart disease in individuals with diabetes: the atherosclerosis risk in communities (ARIC) study. Diabetes 51: 3524-3531.

16. Malpas SC, Maling TJ (1990) Heart-rate variability and cardiac autonomic function in diabetes. Diabetes 39: 1177-1181.
17. Pagani M, Malfatto G, Pierini S, Casati R, Masu AM, et al. (1988) Spectral analysis of heart rate variability in the assessment of autonomic diabetic neuropathy. J Auton Nerv Syst 23: 143-153.

18. Acharya UR, Vidya KS, Ghista DN, Lim WJE, Molinari F, et al. (2015) Computer-aided diagnosis of diabetic subjects by heart rate variability signals using discrete wavelet transform method. Knowledge-Based Systems 81: 56-64.

19. Tarvainen MP, Laitinen TP, Lipponen JA, Cornforth DJ, Jelinek HF (2014) Cardiac autonomic dysfunction in type 2 diabetes - effect of hyperglycemia and disease duration. Front Endocrinol 130: 1-9.

20. Kelarev AV, Stranieri A, Yearwood JL, Abawajy J, Jelinek HF (2012) Improving classifications for cardiac autonomic neuropathy using multilevel ensemble classifiers and feature selection based on random forest. Proceedings of Data Mining and Analytics, AusDM 2012, 11th Australasian Data Mining Conference, CRPIT.

21. Stranieri A, Abawajy J, Kelarev A, Huda S, Chowdhury M, et al. (2013) An approach for Ewing test selection to support the clinical assessment of cardiac autonomic neuropathy. Artif Intell Med 58: 185-193.

22. Abawajy J, Kelarev A, Chowdhury M, Stranieri A, Jelinek HF (2013) Predicting cardiac autonomic neuropathy category for diabetic data with missing values. Comput Biol Med 43: 1328-1333.

23. Kelarev AV, Abawajy J, Stranieri A, Jelinek HF (2013) Empirical investigation of decision tree ensembles for monitoring cardiac complications of diabetes. International Journal of Data Warehousing and Mining 9: 1-18.

24. Jelinek HF, Abawajy JH, Kelarev AV, Chowdhury MU, Stranieri A (2014) Decision trees and multi level ensemble classifiers for neurological diagnostics. AIMS Medical Science 1: 1-12.

25. Jelinek HF, Abawajy JH, Cornforth D, Kowalczyk A, Negnevitsky M, et al. (2015) Multi-layer attribute selection and classification algorithm for the diagnosis of cardiac autonomic neuropathy based on HRV attributes. AIMS Medical Science 2: 396- 409.

26. Abawajy J, Kelarev A, Chowdhury MU, Jelinek HF (2016) Enhancing predictive accuracy of cardiac autonomic neuropathy using blood biochemistry features and iterative multi-tier ensembles. IEEE J. Biomedical Health Informatics 20: 408-415.

27. Ewing DJ, Campbell IW, Clarke BF (1980) The natural history of diabetic autonomic neuropathy. Q J Med 49: 95-108.

28. Ewing DJ, Martyn CN, Young RJ, Clarke BF (1985) The value of cardiovascular autonomic function tests: 10 years experience in diabetes. Diabetes Care 8: 491-498.

29. Teich MC, Lowen SB, Jost BM, Vibe-Rheymer K (2000) Heart rate variability: measures and models pp: 1-84.

30. Zaccardi F, Khan H, Laukkanen JA (2014) Diabetes mellitus and risk of sudden cardiac death: a systematic review and meta-analysis. Int J Cardiol 177: 535-537.

31. Karmakar C, Jelinek H, Khandoker A, Tulppo M, Makikallio T, Kiviniemi A, et al. (2012) Identifying increased risk of post-infarct people with diabetes using multi-lag tone-entropy analysis. Conference proceedings: Annual International Conference of the IEEE Engineering in Medicine and Biology Society.

32. Acharya UR, Tong J, Subbhuraam VS, Chua CK, Ha TP, et al. (2012) Computer-based identification of type 2 diabetic subjects with and without neuropathy using dynamic planter pressure and principal component analysis. Journal of Medical Systems 36: 2483-2491.

33. Jelinek HF, Munro J, Tinley P (2010) Dynamic first metatarsophalangeal joint movement in diabetes foot assessment of type 2 diabetic women. J Diab Foot Compl 2: 51-56.

34. Wong TY, Klein R, Klein BE, Tielsch JM, Hubbard L, et al. (2001) Retinal microvascular abnormalities and their relationship with hypertension, cardiovascular disease, and mortality. Survey of Ophthalmology 46: 59-80.

35. Khandoker AH, Jelinek HF, Palaniswami M (2009) Identifying diabetic patients with cardiac autonomic neuropathy by heart rate complexity analysis. BioMedical Engineering OnLine 8: 1-12. 
Citation: Jelinek HF, Cornforth DJ, Kelarev AV (2016) Machine Learning Methods for Automated Detection of Severe Diabetic Neuropathy. J Diabetic Complications Med 1: 108. doi:10.4172/jdcm.1000108

Page 7 of 7

36. Laitinen T, Vauhkonen I, Niskanen L, Hartikainen J, Lansimies E, et al. (1999) Power spectral analysis of heart rate variability during hyperinsulinemia in nondiabetic offspring of type 2 diabetic patients: evidence for possible early autonomic dysfunction in insulin-resistant subjects. Diabetes 48: 1295-1299.

37. Cornforth DJ, Tarvainen MP, Jelinek HF (2013) Using Renyi entropy to detect early cardiac autonomic neuropathy. Conf Proc IEEE Eng Med Biol Soc 2013: 5562-5565.

38. Cornforth D, Tarvainen M, Jelinek HF (2013) Computational intelligence methods for the identification of early cardiac autonomic neuropathy. Proceedings of the 8th IEEE Conference on Industrial Electronics and Applications (ICIEA).

39. Cornforth D, Tarvainen MP, Jelinek HF (2014) Automated selection of measures of heart rate variability for detection of early cardiac autonomic neuropathy. Proceedings of the International Conference Computing in Cardiology Conference (CinC).

40. Cornforth D, Jelinek HF (2007) Automated classification reveals morphological factors associated with dementia. Applied Soft Computing 8: $182-190$.

41. Jelinek HF, Wilding C, Tinley P (2006) An innovative multi-disciplinary diabetes complications screening programme in a rural community: A description and preliminary results of the screening. Am J Public Health 12: $14-20$.

42. Wessel N, Voss A, Malberg H, Ziehmann C, Voss HU, et al. (2000) Nonlinear analysis of complex phenomena in cardiological data Herzschrittmacher Therapie Elektrophysiol 11: 159-173.

43. Ernst G (2013) Heart Rate Variability. Springer, London.

44. Kelarev AV (2003) Graph Algebras and Automata. Marcel Dekker, New York.

45. Kelarev A, Ryan J, Yearwood J (2009) Cayley graphs as classifiers for data mining: The influence of asymmetries. Discrete Mathematics 309: 5360-5369.

46. Hartigan JA, Wong MA (1979) Algorithm AS 136: a k-means clustering algorithm. Journal of the Royal Statistical Society, Series C 28: 100-108.
47. Comaniciu D, Meer P (2002) Mean Shift: a robust approach toward feature space analysis. IEEE Transactions on Pattern Analysis and Machine Intelligence 24: 603-619.

48. Pedregosa F, Varoquaux G, Gramfort A, Michel V, Thirion B, et al. (2011) Scikit-learn: Machine Learning in Python. Journal of Machine Learning Research 12: 2825- 2830.

49. Scikit-learn (2016) Scikit-learn: Machine Learning in Python.

50. Karypis G, Kumar V (1998) METIS: A software package for partitioning unstructured graphs, partitioning meshes, and computing fill-reducing orderings of sparse matrices; University of Minnesota, Department of Computer Science and Engineering, Army HPC Research Centre, Minneapolis, Technical Report.

51. Zhang T, Ramakrishnan R, Livny M (1996) BIRCH: an efficient data clustering method for very large databases". Proceedings of the 1996 ACM SIGMOD international conference on management of data (SIGMOD'96).

52. Ester M, Kriegel HP, Sander J, Xu X (1996) A density-based algorithm for discovering clusters in large spatial databases with noise. Proceedings of the Second International Conference on Knowledge Discovery and Data Mining (KDD-96). AAAI Press.

53. Ward JH Jr. (1963) Hierarchical grouping to optimize an objective function. Journal of the American Statistical Association 58: 236-244.

54. Fern XZ, Brodley CE (2004) Solving cluster ensemble problems by bipartite graph partitioning. 21st International Conference on Machine Learning, ICML 04; New York, NY, USA.

55. Yearwood J, Webb D, Ma L, Vamplew P, Ofoghi B, et al. (2009) Applying clustering and ensemble clustering approaches to phishing profiling. Proceedings of the 8th Australasian Data Mining Conference, AusDM2009, CRPIT 101: 25-34.

56. Witten IH, Frank E, Hall MA (2011) Data Mining: Practical Machine Learning Tools and Techniques. (3rd Edsn). Morgan Kaufmann; New York, Sydney.

57. Jelinek HF, Cornforth DJ, Blackmore K (2011) Visualisation in biomedicine as a means of data evaluation. J Visualization 14: 353-359. 